ORIGINAL PAPER

\title{
The utility of glomerular C4D immunostaining \\ IN RENAL BIOPSIES IN PATIENTS WITH IMMUNOGLOBULIN \\ A nephropathy. A Clinicopathological STUDY
}

\author{
MaŁgorzata Wągrowska-Danilewicz ${ }^{1}$, Marian DanilewicZ ${ }^{2}$
}

${ }^{1}$ Department of Nephropathology, Medical University of Lodz, Poland

${ }^{2}$ Department of Pathomorphology, Medical University of Lodz, Poland

\begin{abstract}
The course of $\operatorname{IgA}$ nephropathy (IgAN) is highly variable and ranges from a totally benign condition to end-stage renal disease in approximately one third of cases. The identification of new prognostic markers could provide insights into the pathogenesis of IgAN and unveil new therapeutic avenues. Glomerular deposition of C4d is a marker of activation of the lectin pathway of complement. It is thought that activation of the lectin pathway in $\operatorname{IgAN}$ is associated with more severe renal damage, and more severe histological findings.

In view of the above, the aim of the present study was to compare the clinical presentation, laboratory data, and histological lesions in the renal biopsy in IgAN patients with positive and negative staining for mesangial C4d depositions.

Our study revealed that hypertension, severe proteinuria, a high level of serum creatinine, low eGFR at the time of presentation, as well as tubular atrophy/interstitial fibrosis $>50 \%$, and endocapillary proliferation were significantly more frequent in the $\mathrm{C} 4 \mathrm{~d}(+)$ group than in the $\mathrm{C} 4 \mathrm{~d}(-)$ group. Based on our research, we can assume that mesangial immunoexpression of $\mathrm{C} 4 \mathrm{~d}$ seems to be a useful prognostic factor in $\operatorname{IgAN}$.
\end{abstract}

Key words: IgA nephropathy, C4d staining, outcome of IgA nephropathy.

\section{Introduction}

$\operatorname{Ig} A$ nephropathy $(\operatorname{Ig} A N)$ is the most common primary glomerulonephritis worldwide [1], and it accounts for about $20 \%$ of biopsies performed for glomerular disease in Poland [2]. This glomerulopathy presently can only be diagnosed by immunohistological examination of a kidney biopsy [3]. Although the light-microscopic pattern of $\operatorname{IgA}$ nephropathy may vary widely, it is usually characterised by mesangioproliferative changes in glomeruli with deposition of $\operatorname{IgA}$ [4]. Mesangial IgA deposition induces an inflammatory process that involves mesangial proliferation, interstitial damage, and proteinuria [5]. Activation of complement plays a key role in the pathogenesis of IgAN $[6,7]$. Complement activation runs through three pathways: classic, alternative, and mannose-binding lectin. Recent data have revealed the ability of polymeric $\operatorname{IgA}$ to activate the lectin pathway $[5,8,9,10]$. The course of $\operatorname{Ig} A$ nephropathy is highly variable and ranges from a totally benign condition [11] to end-stage renal disease in approximately one third of cases $[5,12,13]$. Some clinical and histological factors, such as arterial hypertension, proteinuria, renal function, and Oxford classification score, determine the final outcome of patients with IgAN [14]. The identification of new prognostic markers could provide insights into pathogenesis of IgAN and unveil new therapeutic avenues [15]. Glomerular deposition of $\mathrm{C} 4 \mathrm{~d}$ is a marker of activation of 
the lectin pathway of complement [5]. It is thought that activation of the lectin pathway in $\operatorname{IgAN}$ is associated with more severe renal damage, and more severe histological findings $[5,15,16]$.

In view of the above, the aim of the present study was to compare the clinical presentation, laboratory data, and histological lesions in the renal biopsy in IgAN patients with positive and negative staining for mesangial C4d depositions.

\section{Material and methods}

Material consists of renal biopsy specimens obtained for diagnostic purposes from 43 adult patients (29 males and 14 females) with IgA nephropathy, who had undergone renal biopsy between 2010 and 2015 in the Nephropathology Centre, Medical University of Lodz. The patients' age, gender, presence of hypertension, nephrotic syndrome, urinary protein excretion level, haematuria, serum creatinine level, and glomerular filtration rate (eGFR) were noted at the time of the renal biopsy. The renal biopsy specimens were routinely processed by light microscopy and immunofluorescence (IF). Criteria applied to the biopsy specimens were as follows: minimal number of 10 non-sclerosed glomeruli for light microscopy, and a minimal number of five non-sclerosed glomeruli for immunofluorescence study. The samples were embedded in paraffin and sectioned at $2 \mu \mathrm{m}$, followed by haematoxylin and eosin (HE), Masson's trichrome, periodic acid-Schiff, methenamine-silver, and Congo red staining. For IF the samples were sectioned in frozen conditions, followed by a direct staining for IgG (DAKO, polyclonal rabbit anti-human IgG/FITC antibody, dilution 1 : 10), IgA (DAKO, polyclonal rabbit anti-human IgA/FITC antibody, dilution 1 : 10), IgM (DAKO, polyclonal rabbit anti-human IgM/FITC antibody, dilution 1 : 10), C3 (DAKO polyclonal rabbit anti-human $\mathrm{C} 3 \mathrm{c}$ Complement/

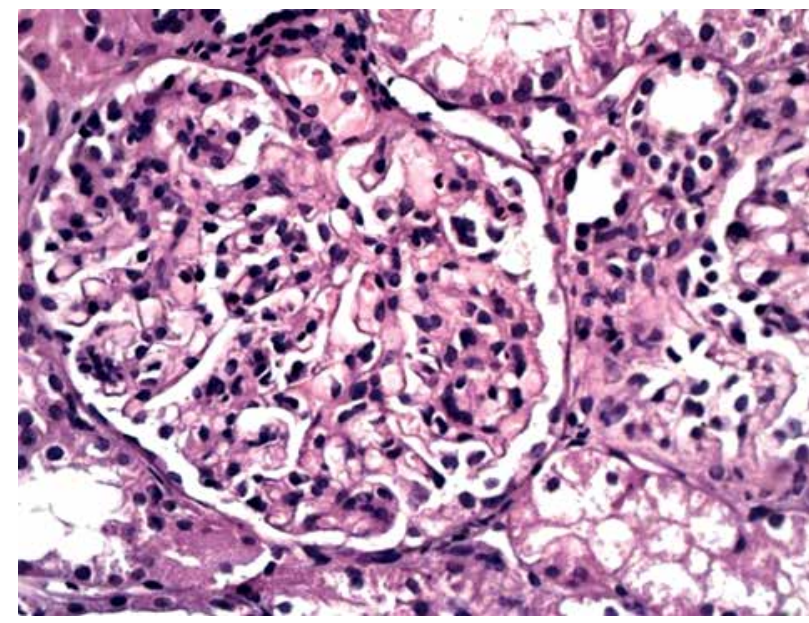

Fig. 1. Mesangial proliferative lesions in the renal tissue in patient with IgAN C4d (-). HE staining, magn. $200 \times$
FITC antibody, dilution 1 : 20), C1q (DAKO, rabbit anti-human C1q Complement/FITC antibody, dilution $1: 20), \kappa$ light chain (DAKO, polyclonal rabbit anti-human $\kappa$ light chains/FITC antibody, dilution $1: 10$ ), and $\lambda$ light chain (DAKO, polyclonal rabbit-anti human $\lambda$ light chains/FITC antibody, dilution 1 : 10). For $\mathrm{C} 4 \mathrm{~d}$ indirect immunofluorescence method was performed: murine monoclonal anti-human C4d antibody, Quidel, diluted 1 : 50, followed by DAKO polyclonal rabbit anti-mouse immunoglobulin/FITC antibody diluted 1 : 10. All sections were mounted in aqueous medium and examined under a dark field ultraviolet fluorescence microscope (Olympus BX41). Negative controls were carried out by incubation slides with PBS in the absence of the primary antibody, and always yielded negative results.

In immunofluorescence findings the localisation of immune deposits, the intensity of immunoglobulin, components of complement staining, $\kappa$ and $\lambda$ light chain staining, and the class of immunoglobulin in deposits were evaluated. A semiquantitative assessment of the intensity of staining was given as: lack of staining $(0)$, mild $(+1)$, moderate $(+2)$, and strong $(+3)$.

Histological lesions were classified using Oxford pathologic classification criteria [14]. In the light microscopy the presence of mesangial proliferation - M (Fig. 1), endocapillary proliferation - E (Fig. 2), and segmental glomerular sclerosis - S (Fig. 3) and the severity of tubular atrophy/interstitial fibrosis $-\mathrm{T}$ (Fig. 4) were noted. On the basis of positive or negative mesangial $\mathrm{C} 4 \mathrm{~d}$ staining the studied material was classified into IgAN C4d (+) group or IgAN C4d (-) group.

Statistical analysis using Statistica 8 software was performed to assess the differences in the clinical presentation, laboratory data, and histological renal damage between both IgAN groups. The differences between groups were assessed using Mann-Whitney

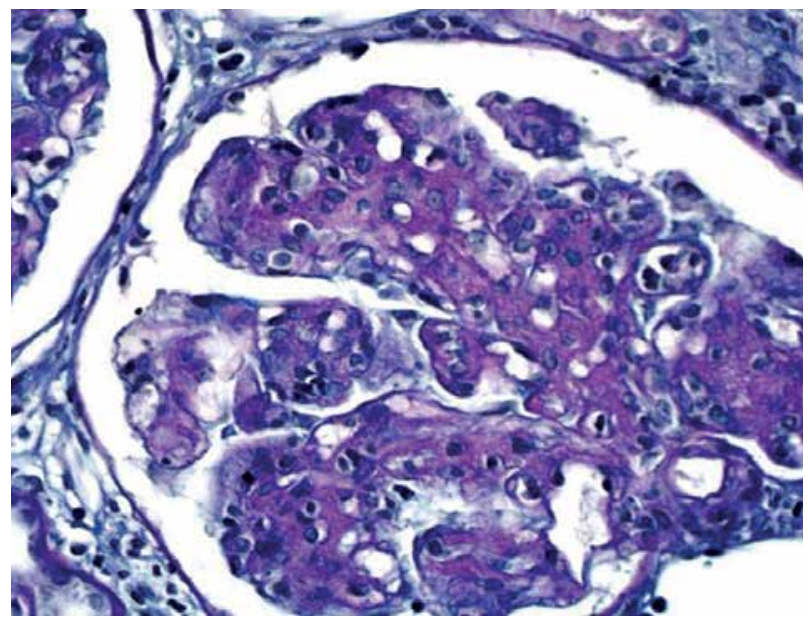

Fig. 2. Endocapillary proliferative lesions in the renal tissue in patient with IgAN C4d (+). PAS staining, magn. $200 \times$ 


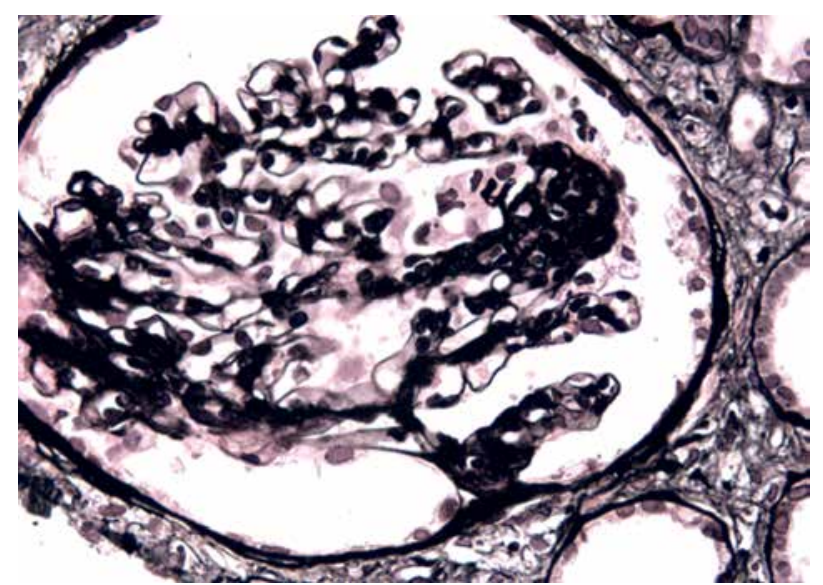

Fig. 3. Segmental glomerular sclerosis in the renal tissue in patient with IgAN C4d (+). Methenamine-silver staining, magn. $200 \times$

$\mathrm{U}$ test and univariate $\chi^{2}$ test. Results were considered statistically significant if $\mathrm{p}<0.05$.

\section{Results}

Dominant or codominant granular IgA mesangial staining was seen in all biopsies (Fig. 5). The immunostaining for IgG and IgM was negative in all studied renal tissue specimens. The granular mesangial immunostaining for $\kappa$ and $\lambda$ light chains was seen in all biopsies.

Of 43 patients with $\operatorname{IgAN}, 11$ were classified as C4d-positive, and 32 as $\mathrm{C} 4 \mathrm{~d}$-negative. In theC $4 \mathrm{~d}$-positive group mesangial pattern of $\mathrm{C} 4 \mathrm{~d}$ staining was seen (Fig. 6). C1q staining was negative in all of the patients. Strong to moderate mesangial granular immunostaining for C3 was observed in all biopsies. The IgAN C4d-positive group consisted of eight males and three females (mean age $37 \pm 9$ years). In the IgAN C4d-negative group there were 21 males and 11 females (mean age $33 \pm 11$ years). Haematu-

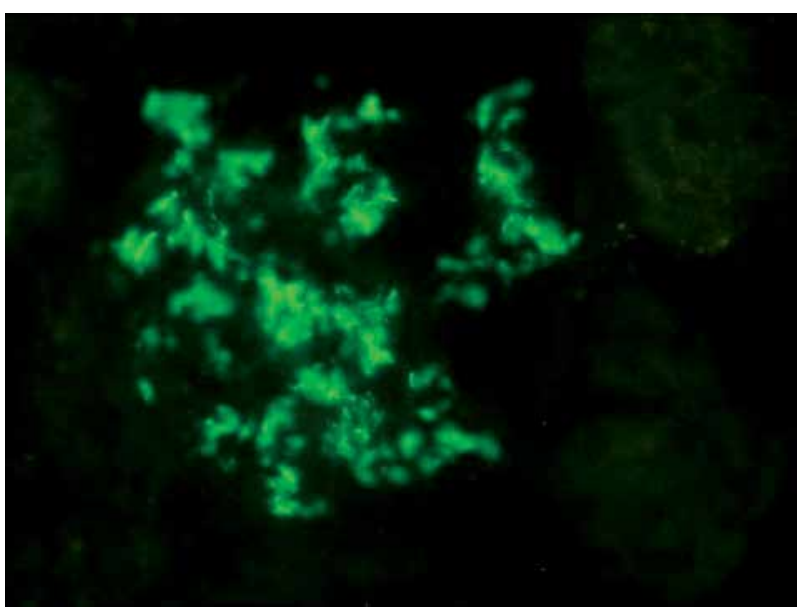

Fig. 5. IF. Strong granular mesangial IgA immunostaining in the renal tissue in patients with IgAN, magn. $200 \times$

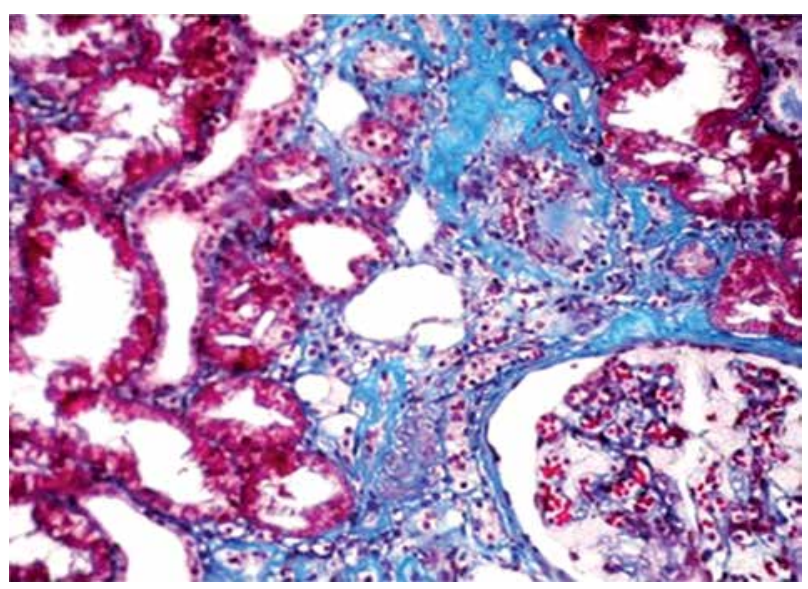

Fig. 4. Interstitial fibrosis and tubular atrophy in the renal tissue in patient with IgAN C4d (+). Masson's trichrome staining, magn. $100 \times$

ria was noted in all patients in both studied groups. Clinical and laboratory data in the IgAN C4d-positive and IgAN C4d-negative groups are shown in Table I. The proportion of patients with hypertension was significantly higher $(72.7 \%$ vs. $37.5 \%, \mathrm{p}<0.001)$ in C4d-positive patients. At the time of presentation, C4d-positive patients had a significantly lower eGFR $(63.6 \%$ vs. $21.8 \%, \mathrm{p}<0.001)$, a higher amount of proteinuria $(45.4 \%$ vs. $18.7 \%, \mathrm{p}<0.002)$, and a higher serum creatinine level $(63.6 \%$ vs. $25 \%$, p $<$ 0.001), compared with C4d-negative patients.

Histological findings are shown in Table II. Histological examination of renal biopsy specimens in the C4d-positive group revealed mesangial proliferation in more than $50 \%$ glomeruli (M1, according to the Oxford classification) in nine patients $(81.8 \%)$ and in $27(84.4 \%)$ C4d-negative patients ( $\mathrm{p}=0.76, \mathrm{NS})$. Endocapillary proliferation (E1, according to the Oxford classification) was noted in seven C4d-positive $(63.6 \%)$ and in $10 \mathrm{C} 4 \mathrm{~d}$-negative patients $(31 \%)(\mathrm{p}<$ 0.001). Segmental glomerular sclerosis (S1, accord-

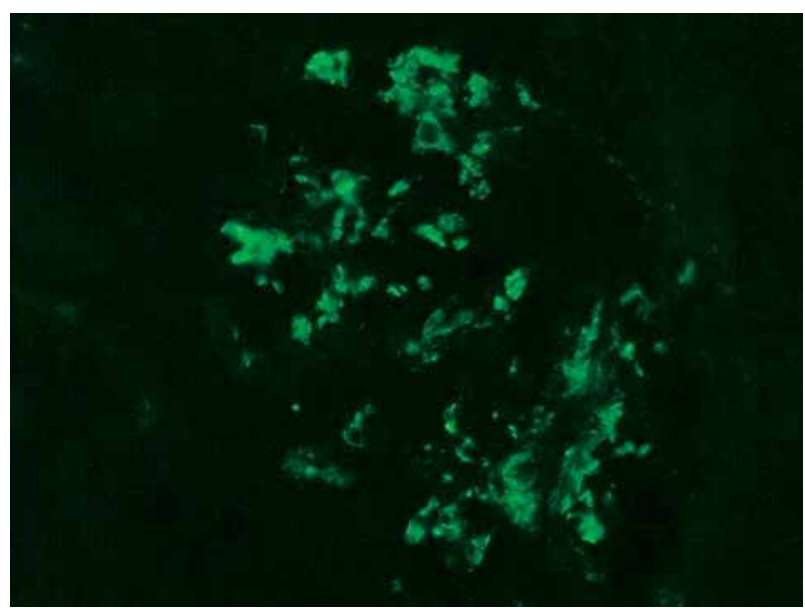

Fig. 6. IF. Mesangial immunostaining for $\mathrm{C} 4 \mathrm{~d}$ in the renal tissue in patient with IgAN C4d- positive group, magn. $200 \times$ 
Table I. Clinical and laboratory data in IgAN C4d (+) and IgAN C4d (-) groups (number of patients, \%)

\begin{tabular}{lcccc}
\hline Groups & $\begin{array}{c}\text { HyPERTENSION } \\
(>140 / 90)\end{array}$ & $\begin{array}{c}\text { ProteInURIA } \\
(>3 \mathrm{G} / 24 \mathrm{H})\end{array}$ & $\begin{array}{c}\text { SERUM CREATININE } \\
(>1.4 \mathrm{MG} / \mathrm{DL})\end{array}$ & $\begin{array}{c}\text { EGFR } \\
(<59 \mathrm{ML} / \mathrm{MIN})\end{array}$ \\
\hline $\begin{array}{l}\text { C4d-positive } \\
(\mathrm{n}=11)\end{array}$ & $8(72.7 \%)$ & $5(45.4 \%)$ & $7(63.6 \%)$ & $7(63.6 \%)$ \\
\hline $\begin{array}{l}\mathrm{C} 4 \mathrm{~d}-\text {-negative } \\
(\mathrm{n}=32)\end{array}$ & $12(37.5 \%)$ & $6(18.7 \%)$ & $8(25 \%)$ & $7(21.8 \%)$ \\
\hline P value & $<0.001$ & $<0.002$ & $<0.001$ & $<0.001$ \\
\hline
\end{tabular}

Table II. Histologic findings in C4d-positive and C4d-negative patients (number of patients, \%)

\begin{tabular}{|c|c|c|c|c|}
\hline GROUPS & $\begin{array}{c}\text { MESANGIAL PROLIFERATION } \\
\text { M1 }\end{array}$ & $\begin{array}{c}\text { ENDOCAPILLARY } \\
\text { PROLIFERATION E1 }\end{array}$ & $\begin{array}{l}\text { SEGMENTAL GLOMERULAR } \\
\text { SCLEROSIS S1 }\end{array}$ & $\begin{array}{c}\text { TUBULAR } \\
\text { ATROPHY T2 }\end{array}$ \\
\hline $\begin{array}{l}\text { C4d-positive } \\
(\mathrm{n}=11)\end{array}$ & $9(81.8 \%)$ & $7(63.6 \%)$ & $8(72.7 \%)$ & $6(54.5 \%)$ \\
\hline $\begin{array}{l}\text { C4d-negative } \\
(\mathrm{n}=32)\end{array}$ & $27(84.4 \%)$ & $10(31 \%)$ & $24(75 \%)$ & $5(15.6 \%)$ \\
\hline $\mathrm{P}$ value & 0.76 (NS) & $<0.001$ & 0.79 (NS) & $<0.001$ \\
\hline
\end{tabular}

ing to the Oxford classification) was seen in eight C4d-positive patients $(72.7 \%)$ and in 24 from the C4d-negative group $(75 \%)(\mathrm{p}=0.79, \mathrm{NS})$. Tubular atrophy/interstitial fibrosis (T2, according to the Oxford classification) was present in six C4d-positive $(54.5 \%)$ and in five C4d-negative patients $(15.6 \%)$ $(\mathrm{p}<0.001)$.

\section{Discussion}

The variability in the clinical course of IgAN justifies efforts to determine clinical and histological features that predict the development of renal failure in this glomerulopathy, and thus to guide therapy. Recently mesangial deposition of $\mathrm{C} 4 \mathrm{~d}$ is regarded as a prognostic factor in $\operatorname{IgAN}$ [5, 15, 16]. Roos et al. [5] revealed that patients with IgAN are divided into two groups on the basis of the pattern of complement activation. In the patients with negative glomerular staining for mannose-binding lectin (C4d-negative) the activation of the complement occurs via the alternative pathway. When these stainings are positive (C4d-positive), activation of complement occurs via the lectin pathway [5]. A deeper understanding of the mechanisms of complement activation may help to elucidate the pathogenesis of IgA nephropathy [8].

In our study the $\mathrm{C} 4 \mathrm{~d}$-positive group comprised $25.6 \%$ of $\operatorname{IgAN}$ patients whereas for the C4d-negative group it was $74.4 \%$. In the study by Espinosa et al. [16] C4d-positive patients were more numerous: $32.2 \%$ and $67.8 \%$, respectively. In the latter study by Espinosa et al. [15] these percentages were 38.5\% and $61.5 \%$, respectively. Similarly to our results,
Roos et al. [5] found $25 \%$ of IgAN patients to be C4d-positive and $75 \%$ C4d-negative.

Analysing the clinical data, we found, similarly to others $[15,16]$, that the number of patients with hypertension was significantly higher in C4d-positive patients as compared to $\mathrm{C} 4 \mathrm{~d}$-negative individuals. The urinary protein excretion in our IgAN cases was also significantly more frequent in C4d-positive participants. In an earlier work by Espinosa et al. [16] this difference was not significant; however, in the latter study, [15] on a larger cohort of IgAN patients, they revealed statistical significance. Of note, in the study by Sahin et al. this parameter also differed significantly in C4d-positive and C4d-negative groups [17]. At the time of presentation, our C4d-positive patients had a significantly lower eGFR and a higher serum creatinine level compared with C4d-negative patients. These findings are in concordance the observations of other authors [8, 15, 16]. Interestingly, in the study by Shu et al. all these parameters were in IgAN patients with worse prognosis, similarly to our C4d-positive group, and differed significantly as compared to the IgAN group, with better prognosis [18].

The comparison of histological findings between the $\mathrm{C} 4 \mathrm{~d}$-positive and $\mathrm{C} 4 \mathrm{~d}$-negative groups revealed that mesangial proliferation in $>50 \%$ glomeruli (M1, according to the Oxford classification) did not differ significantly. This observation is in an agreement with earlier studies $[15,16]$. It should be noted, however, that the results of Sahin et al. [17] showed a highly significant difference of mesangial proliferation in these groups. On the other hand, we noted significant difference as regard endocapillary proliferation 
(E1, according to the Oxford classification). This proliferation was significantly greater in C4d-positive patients as compared to the $\mathrm{C} 4 \mathrm{~d}$-negative group. In contrast, in the study by Espinosa $e t$ al. this difference did not reach statistical significance [15]. It is noteworthy, however, that in the study of these authors endocapillary proliferation was also more frequent in C4d-positive participants. In the present study segmental glomerular sclerosis (S1, according to the Oxford classification) did not differ significantly in the C4d-positive and C4d-negative groups. in contrast to our findings, other authors revealed significant differences in this parameter $[15,17]$, whereas Roos et al. [5] received results similar to ours. Tubular atrophy/interstitial fibrosis (T2, according to the Oxford classification) was significantly more frequent in our C4d-positive group in comparison with C4d-negative patients. This finding is in concordance with other authors $[16,17]$.

In summary, our study revealed that hypertension, severe proteinuria, a high level of serum creatinine, low eGFR at the time of presentation, as well as tubular atrophy/interstitial fibrosis $>50 \%$ (T2), and endocapillary proliferation (E1) were significantly more frequent in the C4d-positive group than in the C4d-negative group. Based on our research, we can assume that mesangial immunoexpression of $\mathrm{C} 4 \mathrm{~d}$ seems to be a useful prognostic factor in IgAN.

This work was supported by a grant of the Medical University of Lodz - 503/6-038-01/503-01.

The authors declare no conflict of interest.

\section{References}

1. Wyatt R, Julian B. IgA nephropathy. N Eng J Med 2013; 368: 2402-2414.

2. Perkowska-Ptasińska A, Bartczak A, Wągrowska-Danilewicz M, et al. Chronic kidney disease. Clinical epidemiology - 2. Nephrol Dial Transplant 2015; 30: suppl. 3.

3. Sethi S, Haas M, Markowitz GS et al. Mayo Clinic/Renal Pathology Society consensus report on pathologic classification, diagnosis and reporting of GN. J Am Soc Nephrol 2015; 27: $1-7$.

4. Floege J, Moura IC, Daha MR. New insight into the pathogenesis of IgA nephropathy. Semin Immunopathol 2014; 36: 431-442.

5. Roos A, Rastaldi MP, Calvaresi N, et al. Glomerular activation of the lectin pathway of complement in IgA nephropathy is associated with more severe renal disease. J Am Soc Nephrol 2006; 17: 1724-1734.

6. Daha MR, van Kooten C. Role of complement in IgA nephropathy. J Nephrol 2016; 29: 1-4.

7. Maillard N, Wyatt RJ, Julian BA, et al. Current understanding of the role of complement in IgA nephropathy. J Am Soc Nephrol 2015; 26: 1503-1512.

8. Ohsawa I, Ishii $\mathrm{M}$, Ohi $\mathrm{H}$, et al. Pathological scenario with mannose-binding lectin in patients with $\operatorname{IgA}$ nephropathy. J Biomed Biotechnol 2012; 476739: 1-5.
9. Liu LL, Liu N, Chen Y, et al. Glomerular mannose-binding lectin deposition is a useful prognostic predictor in immunoglobulin A nephropathy. Clin Exp Immunol 2013; 174: 152-160.

10. Shi B, Wang L, Mou S, et al. Identification of mannose-binding lectin as a mechanism in progressive immunoglobulin A nephropathy. Int J Clin Exp Pathol 2015; 8: 1889-1899.

11. Gutiérrez E, Zamora I, Ballarín JA, et al. Long-term outcomes of IgA nephropathy presenting with minimal or no proteinuria. J Am Soc Nephrol 2012; 23: 1753-1760.

12. Donadio JV, Grande JP. IgA nephropathy. N Engl J Med 2002; 347: 738-748.

13. Le W, Liang S. Hu Y, et al. Long-term renal survival and related risk factors in patients with $\operatorname{IgA}$ nephropathy: results from a cohort of 1155 cases in a Chinese adult population. Nephrol Dial Transplant 2012; 27: 1479-1485.

14. Roberts IS, Cook HT, Troyanov S, et al. The Oxford classification of IgA nephropathy: Pathology definitions, correlations, and reproducibility. Kidney Int 2009; 76: 546-556.

15. Espinosa M, Ortega R, Sanchez M, et al. Association of C4d deposition with clinical outcomes in IgA nephropathy. Clin J Am Soc Nephrol 2014; 9: 897-904.

16. Espinosa M, Ortega R, Gómez-Carrasco JM, et al. Mesangial C4d deposition: a new prognostic factor in IgA nephropathy. Nephrol Dial Transplant 2009; 24: 886-889.

17. Sahin OZ, Yavas H, Tasli F, et al. Prognostic value of glomerular C4d staining in patients with IgA nephritis. Int J Clin Exp Pathol 2014; 7: 3299-3304.

18. Shu D, Xu F, Su Z, et al. Risk factors of progressive IgA nephropathy which progress to end stage renal disease within ten years: a case-control study. BMC Nephrol 2017; 18: 1-6.

\section{Address for correspondence}

Małgorzata Wągrowska-Danilewicz

Department of Nephropatology Medical University of Lodz Pomorska 251

92-216 Łódź, Poland

tel./fax +48426757633

e-mail: malgorzata.wagrowska-danilewicz@umed.lodz.pl 